\title{
O campo Saúde do Trabalhador nos 25 anos da Revista Ciência \& Saúde Coletiva
}

\author{
Worker's Health in the 25 years of the Journal Ciência \& Saúde \\ Coletiva
}

Francisco Antonio de Castro Lacaz (https://orcid.org/0000-0001-7621-3756) ${ }^{1}$ Patrícia Martins Goulart (https://orcid.org/0000-0002-5080-9241) ${ }^{2}$

Edvânia Ângela de Souza (https://orcid.org/0000-0002-8997-7592) 3

Carla Andrea Trapé (https://orcid.org/0000-0002-3272-6565) 4

Dimitri Moita (https://orcid.org/0000-0002-1814-7751) ${ }^{5}$

Gabriela Mota-Sousa (https://orcid.org/0000-0002-6710-773X) ${ }^{6}$

Bruno Chapadeiro Ribeiro (https://orcid.org/0000-0003-0167-0164) ${ }^{7}$
${ }^{1}$ Escola Paulista de

Medicina, Universidade Federal de São Paulo (UNIFESP). R. Botucatu 740, Vila Clementino. 04023-062 São Paulo SP Brasil.

franlacaz@hotmail.com

${ }^{2}$ Instituto Saúde e

Sociedade, UNIFESP. Santos

SP Brasil.

${ }^{3}$ Faculdade de Ciências

Humanas e Sociais,

Universidade Estadual de

São Paulo. São Paulo SP

Brasil.

${ }^{4}$ Escola de Enfermagem,

Universidade de São Paulo.

São Paulo SP Brasil.

${ }^{5}$ Programa de Pós-

Graduação em Psicologia,

Universidade Federal do

Ceará. Fortaleza CE Brasil.

${ }^{6}$ Programa de Pós-

Graduação em Saúde

Coletiva, Escola Paulista de

Medicina, UNIFESP. São

Paulo SP Brasil.

${ }^{7}$ Programa de Pós-

Graduação em Psicologia

da Saúde, Universidade

Metodista de São Paulo. São

Bernardo do Campo SP

Brasil.

\begin{abstract}
This paper addresses Worker's Health scientific production, specifically about how the Journal Ciência \& Saúde Coletiva has contributed to the consolidation of this field. This is a qualitative and descriptive study based on a review of the Journal's collection from 1996 to 2019. We selected a final sample of 156 texts organized by thematic groups. The theme was designed with the predominance of Epidemiological and Public Policy studies, studies of a theoretical-methodological nature and related to World of Work Transformations. Approximately $60 \%$ of publications are concentrated in the 2009-2015 period. A significant decline in the number of works was observed as of 2016. The papers address Worker's Health in a comprehensive and diversified manner. There is a greater emphasis on research on work and environment, health issues, formulation and implementation of policies and programs. The journal significantly contributes to the national Worker's Health. Strengthening academic production in this field remains a challenge, especially in the face of the loss of rights.
\end{abstract}

Key words Worker's health, Work and health, Work and environment, Worker's health surveillance
Resumo Este artigo trata da produção cientifica relativa ao campo Saúde do Trabalhador, mais especificamente, sobre como a Revista Ciência \& Saúde Coletiva, tem contribuído para a consolidação deste. Trata-se de um estudo qualitativodescritivo com base em uma revisão do acervo da revista, no período de 1996 a 2019. Efetivou-se a seleção de artigos, resultando na amostra de 156 textos, organizada por grupos temáticos. O tema foi se desenhando com a predominância de estudos Epidemiológicos e de Políticas Públicas, e de caráter Teórico-metodológicos e relacionados às Transformações no Mundo do Trabalho. Cerca de $60 \%$ das publicações estão concentradas entre os anos de 2009 e 2015. Há diminuição relevante do número de publicações a partir de 2016. Os artigos abordam o campo Saúde do Trabalhador de maneira abrangente e diversificada. Há maior ênfase em investigações sobre trabalho e ambiente, agravos à saúde, formulação e implementação de politicas e programas. A revista cumpre expressiva contribuição ao campo Saúde do Trabalhador no cenário nacional. Fortalecer a produção acadêmica neste campo, especialmente diante da derrocada de direitos, é um desafio que se mantém.

Palavras-chave Saúde do trabalhador, Trabalho e saúde, Trabalho e ambiente, Vigilância em saúde do trabalhador 


\section{Introdução}

A importância da discussão sobre o campo Saúde do Trabalhador (ST) vincula-se aos estudos sobre sua incorporação pelo Sistema Único de Saúde (SUS), à ação sindical em sua constituição e à produção acadêmica que lhe deu sustentação $0^{1,2}$. Trata-se de um processo de construção contínua, como aponta Minayo-Gomez ${ }^{3}$.

Os móveis teóricos que subsidiam o discurso teórico e prático da ST surgiram da necessidade imprescindível de avançar no debate da relação trabalho e saúde produzido pela Medicina do Trabalho (MT) e pela Saúde Ocupacional (SO). O fio condutor foi a incorporação das abordagens da Medicina Social Latino-Americana e da Saúde Coletiva $^{1,4,5}$. Apesar de cotejar as formulações da SO, a abordagem da ST, do ponto de vista acadêmico, político e institucional, ultrapassa aquelas análises restritas ao paradigma monocausal, que situa a doença e um agente específico; ou multicausal, no qual a doença se associa a um grupo de fatores de risco (físicos, químicos, biológicos e mecânicos) presentes no ambiente de trabalho ${ }^{1,6}$.

A abordagem desenvolvida pelo campo se ancora na apropriação da categoria processo de trabalho em sua relação com a saúde. $O$ conceito processo de trabalho, a partir da crítica da economia política, compreende o modo de produção, gestão e organização do trabalho, que por meio da intervenção humana e por intermédio dos meios de produção age consciente e intencionalmente, com vistas a obter um determinado resultado, um produto material ou imaterial ${ }^{7}$. Na sociedade capitalista, o processo de trabalho está voltado para atender o objetivo da acumulação (processo de valorização) e seu meio (processo laboral) com um modo específico de trabalho-desgaste e de enfrentamento de classe, que por sua vez determinam um padrão particular de reprodução. Este, ao se combinar com o desgaste, se constitui em um processo de saúde-doença específico para cada grupo social, provoca desgaste da força de trabalho que se expressa em acidentes e doenças ${ }^{8}$.

No que se refere à produção acadêmica, destacam-se autores filiados à Medicina Social Latino -Americana como Laurell ${ }^{9,10}$, Laurell e Noriega ${ }^{8}$ e outros que conceberam a proposta do Modelo Operário Italiano (MOI), desenvolvido na Itália nos anos $1960-70^{11}$, os quais influenciaram tanto a ação sindical, como os serviços de saúde ${ }^{1}$.

Neste percurso, a ação sindical potencializou o desenvolvimento do campo ST, especialmente, na reivindicação, gestão e participação/controle social de trabalhadores nos Programas de Saúde do Trabalhador (PST), que posteriormente passaram a integrar os Centros de Referência em Saúde do Trabalhador (CEREST). A criação do Departamento Intersindical de Estudos e Pesquisas de Saúde e Ambientes de Trabalho (Diesat), em 1980, contribuiu de forma importante para subsidiar essa ação ${ }^{12}$.

Do ponto de vista das políticas de saúde, a ST é um campo de intervenção e práticas desenvolvidas na esfera da saúde pública ${ }^{4,5}$. Assim, a ST adota os princípios do Sistema Único de Saúde (SUS), originado do Movimento de Reforma Sanitária Brasileiro (MRSB), a saber: o direito universal à saúde; a atenção integral, com ênfase para a prevenção e promoção da saúde; as ações descentralizadas e a participação social. O conceito ampliado de saúde, também é um dos fundamentos do SUS, necessário à transformação de práticas sociais para muito além da assistência à saúde.

A partir desses princípios, foi constituída, em 2002, a Rede Nacional de Atenção Integral à Saúde do Trabalhador (Renast), no interior do Ministério da Saúde (MS), como um importante mecanismo legal, inclusive com fonte de financiamento para a organização, a institucionalização e o fortalecimento das ações de ST, no SUS, tanto no nível de assistência e promoção da saúde como de vigilância nos ambientes de trabalho. A RENAST garantiu também a participação dos trabalhadores no acompanhamento dos CEREST articulada à formação e organização dos conselhos gestores, provocando a participação sindical e popular ${ }^{13}$. Em síntese, a criação da RENAST deu origem à Política Nacional de Saúde do Trabalhador (PNST) para o SUS, construída em 2004, que buscou: 1) ampliar sua cobertura para atender todos os trabalhadores; 2) harmonização de normas e articulação das ações; 3 ) precedência da prevenção sobre a reparação; 4) construção da rede integrada de informações em ST; 5) estruturação da formação em ST visando à formação continuada dos trabalhadores que vão operacionalizar a PNST; 6) promoção da agenda integrada de estudos e investigações em $\mathrm{ST}^{13}$.

No ano de 2012, a PNST ganhou uma nova versão, quando foi instituída a Política Nacional de Saúde e Segurança do Trabalhador e da Trabalhadora (PNSTT) ${ }^{14}$, que entre os princípios e as estratégias para a efetivação da ST no SUS, destaca o desenvolvimento da atenção integral à saúde do trabalhador, com ênfase na vigilância, visando à promoção e a proteção da saúde, e a redução da morbimortalidade decorrente dos modelos de desenvolvimento e dos processos produtivos.

Os CEREST e as Vigilâncias Sanitárias, nas três esferas de governo (municipal, estadual e fe- 
deral), vêm desenvolvendo ações e diretrizes para o planejamento e a operacionalização de ações de vigilância nos ambientes de trabalho ou em saúde do trabalhador (VISAT). Vasconcellos et al. ${ }^{15}$ destacam que um dos eixos da PNSTT é a ampliação da influência nos determinantes e condicionantes da saúde relacionados aos processos de trabalho. O CEREST, entendido como suporte às ações de assistência e vigilância, deve "[...] recolher, sistematizar e difundir informações, de modo a viabilizar as ações de vigilância, facilitar os processos de capacitação e educação permanente para os profissionais e técnicos da rede SUS e controle social"16. As ações de VISAT se caracterizam como práticas intersetoriais, articuladas pelo CEREST, que envolvem tanto os serviços do SUS, no caso, as vigilâncias (ambiental, sanitária e epidemiológica), quanto os trabalhadores e representantes sindicais ${ }^{17}$ e, ainda, setores do Ministério do Trabalho e do Ministério Público do Trabalho.

Cabe ainda assinalar a persistência da ocorrência de acidentes e doenças relacionados ao trabalho em níveis elevados, assim como, algumas limitações, por exemplo, no âmbito da política pública, a pequena incorporação pela Atenção Básica $(\mathrm{AB})^{16,18}$ das ações de ST, também as esparsas ações de Educação em ST; retaguarda especializada, bem como ações de Visat ${ }^{19}$.

No âmbito do movimento sindical, a fragilidade em resistir às transformações no mundo do trabalho que envolvem a reestruturação produti$\mathrm{va}^{20} \mathrm{e}$ a desproteção social, marcadas pelas novas formas de contratação, tais como: a terceirização, a subcontratação, o trabalho autônomo, intermitente, em casa (home office), acompanhados do desemprego, além de representar ampla insegurança para a vida, repercutem também na sua capacidade de negociação coletiva ${ }^{21}$ com perdas também para as medidas de saúde e segurança nos ambientes de trabalho e amplo impacto para a saúde mental relacionada ao trabalho ${ }^{22}$.

A complexidade do cenário é notória, como também os desafios que se impõe às pesquisas no campo ST, diante do avanço técnico-científico da computação, da automação industrial avançada, da interatividade nas redes sociais, da internet, dentre outros aspectos presentes nos processos denominados Indústria $4.0^{23}$. Pari passu, se agudizam os constrangimentos vivenciados pelos trabalhadores devido à desproteção social e às novas formas de gestão, condições e organização do trabalho ${ }^{21}$.

Assim, o foco do artigo é apresentar a produção sobre o campo ST publicada no periódico Ciência \& Saúde Coletiva (C\&SC) com dois ob- jetivos: 1) delinear o comportamento das publicações do campo ST no período de 1996 (ano de criação da C\&SC) a 2019;2) apresentar temáticas e abordagens que caracterizam as publicações do campo ST.

\section{Método}

A revisão envolveu a leitura dos títulos de todos os artigos publicados e editoriais dos números temáticos que tratam da ST disponibilizados na homepage da revista C\&SC, no período de 1996 a 2019, excetuando cartas, resenhas e comentários de debatedores.

Primeiramente foram selecionados os artigos relacionados ao campo ST, empregando-se o operador booleano and na associação entre os descritores: Saúde do Trabalhador, Trabalho e Saúde, de um total de 4.928 publicações. Este procedimento visou delinear o comportamento dos artigos naquele período, bem como sua expressão numérica em relação ao total de artigos.

Após leitura dos títulos, palavras-chave e, em alguns casos, dos resumos e textos completos, 291 artigos foram selecionados. Observou-se que vários artigos versavam sobre trabalho e saúde, mas não utilizaram os descritores acima referidos, ou apresentavam os descritores, mas não contemplavam a discussão sob a perspectiva do campo ST, conforme delineado na introdução.

Assim, sem se ater aos descritores, procedeu-se à leitura na íntegra dos resumos e textos completos, por quatro dos sete autores do presente artigo de modo independente, reduzindo a amostra para 263 textos. O critério de exclusão foi a não consideração da relação trabalho e saúde como principal objeto de estudo.

Desse total de 263 artigos, confrontando-se as informações entre os pesquisadores, a amostra se restringiu a 156, sob os mesmos critérios de exclusão mencionados. Por exemplo, um artigo que estudou a questão dos agrotóxicos, porém não os examinava em sua relação com a saúde dos trabalhadores.

A amostra final foi organizada em grupos temáticos (GT), conforme estratégia metodológica adotada por Minayo-Gomez e Thedim-Costa ${ }^{24}$, descrita a seguir.

A abordagem do material constituiu-se inicialmente na elaboração de um panorama que considerasse a natureza dos estudos; os enfoques presentes na formulação e no tratamento dos problemas; as temáticas predominantes; os novos objetos e as tendências teóricas de abor- 
dagem. Frente ao conjunto de conteúdos e temas tratados nos artigos, optou-se por concentrá-los em grupos temáticos capazes de configurar um quadro inicial de análise. Esse processo contou com a avaliação de um pesquisador, que atuou como "juiz" na delimitação do formato final dos GT, a saber: 1) Estudos Epidemiológicos: artigos que apresentam perfis epidemiológicos de agravos à saúde de trabalhadores relacionados com condições, processos e organização do trabalho; 2) Estudos sobre as transformações no mundo do trabalho: artigos que discutem as transformações no mundo do trabalho ocorridas no Brasil a partir de 1990, no contexto do neoliberalismo, que conduziram à reestruturação produtiva, com novos padrões organizacionais e tecnológicos, novas formas de organização social do trabalho e novos métodos denominados 'participativos'; 3 ) Estudos Teórico-metodológicos: artigos que propõem abordagens teóricas e metodológicas ou que criticam abordagens já existentes para a investigação da relação trabalho e saúde; ou aqueles que se propõem a analisar teorias que embasam epistemologicamente o campo. 4) Estudos sobre Políticas Públicas: artigos que têm como objeto específico o estudo de programas governamentais, particularmente suas condições de emergência, seus mecanismos de operação e seus possíveis impactos sobre a ordem social e econômica. Tratam-se de artigos que versam sobre políticas públicas em ST, em seus diversos aspectos, tais como ações intersetoriais, controle social e Visat.

\section{Resultados}

Com o intuito de delinear o comportamento das publicações do campo ST na revista C\&SC, conforme os 4 GT, no período 1996-2019, apresentase, a seguir, as Figuras 1 e 2.

Observa-se que $61 \%$ das publicações analisadas estão concentrados entre os anos de 2009 e 2015 , o dobro da quantidade de textos selecionados nos treze primeiros anos da revista, ou seja, 29\%. A partir de 2016 há uma diminuição das publicações em ST, desde então são apenas 16 artigos, correspondentes a $10 \%$ da amostra. Notese que a C\&CS não apresentou artigos em ST nos anos de 1996, 1997, 1999 e 2007.

A maior frequência de artigos ocorre quando da publicação de números temáticos sobre ST, a saber: número 4 de 2003, dedicado ao debate sobre a integração das ações em ST e saúde ambiental; número 4 de 2005, publicado por ocasião da realização da III Conferência Nacional de Saúde do Trabalhador (3a CNST); número 8 de 2011, que tem por tema saúde, trabalho e ambiente; número 3 de 2013, dedicado à saúde dos profissionais da segurança pública; número 11 de 2013, dedicado à produção de conhecimento em ST; $\mathrm{e}$ número 12 de 2014, dedicado à Visat.

A figura seguinte apresenta um comparativo entre o total de artigos publicados pela C\&SC (excluindo editoriais, cartas, resenhas e comentários de debatedores), representado pela linha, e o percentual que os artigos em ST significam diante do total de artigos a cada ano, mostrado nas colunas.

A representatividade das publicações em ST na C\&SC não acompanha a variação do total de artigos publicados pelo periódico ao longo dos 25 anos. Se em 2003 a produção em ST correspondeu a $15,1 \%$ dos 79 artigos publicados, com o crescimento do número de publicações, o percentual de artigos com a temática ST, ao contrário, vem diminuindo, chegando a 0,7\% em 2019.

Os 156 artigos incluídos nesta análise estão dispostos a partir dos temas que se destacaram nas publicações do campo ST na C\&SC, organizados nos Grupos Temáticos (GTs) apresentados anteriormente.

No grupo temático Estudos Epidemiológicos, foram agrupados 78 artigos, com vertentes teórico-metodológicas e de interpretação distintas. Destes, 37 estudos apresentam investigações sobre trabalhadores submetidos a algum processo de adoecimento, considerando-se variáveis relacionados à antropometria, resultados de exames laboratoriais ou de imagens, ausência ou presença de sintomas do agravo em questão. Fizeram parte desse grupo estudos voltados para o perfil de trabalhadores em diversas ocupações da indústria e dos serviços, além de trabalhos sobre exposição e contaminação por agentes físicos, químicos e biológicos, incluindo aqui trabalho rural e exposição a pesticidas.

Outros 29 trabalhos evidenciaram a multicausalidade do processo de adoecimento em que os agravos estudados foram abordados a partir da perspectiva dos fatores de risco e na interlocução de aspectos biológicos com os psicológicos, sociais e de comportamento individual ${ }^{2,25}$. Parte dos trabalhos apresentava dados sociodemográficos e a relação trabalho e saúde em alguns setores da economia, com destaque para: mineração, agroindústria (sucroalcooleiro, madeireiras), transporte, eletricitários, pescadores e catadores de caranguejo, serviços (bancários, policiais e militares, profissionais da saúde, catadores de lixo), indústria (com destaque para metalúrgicos) entre outros. 


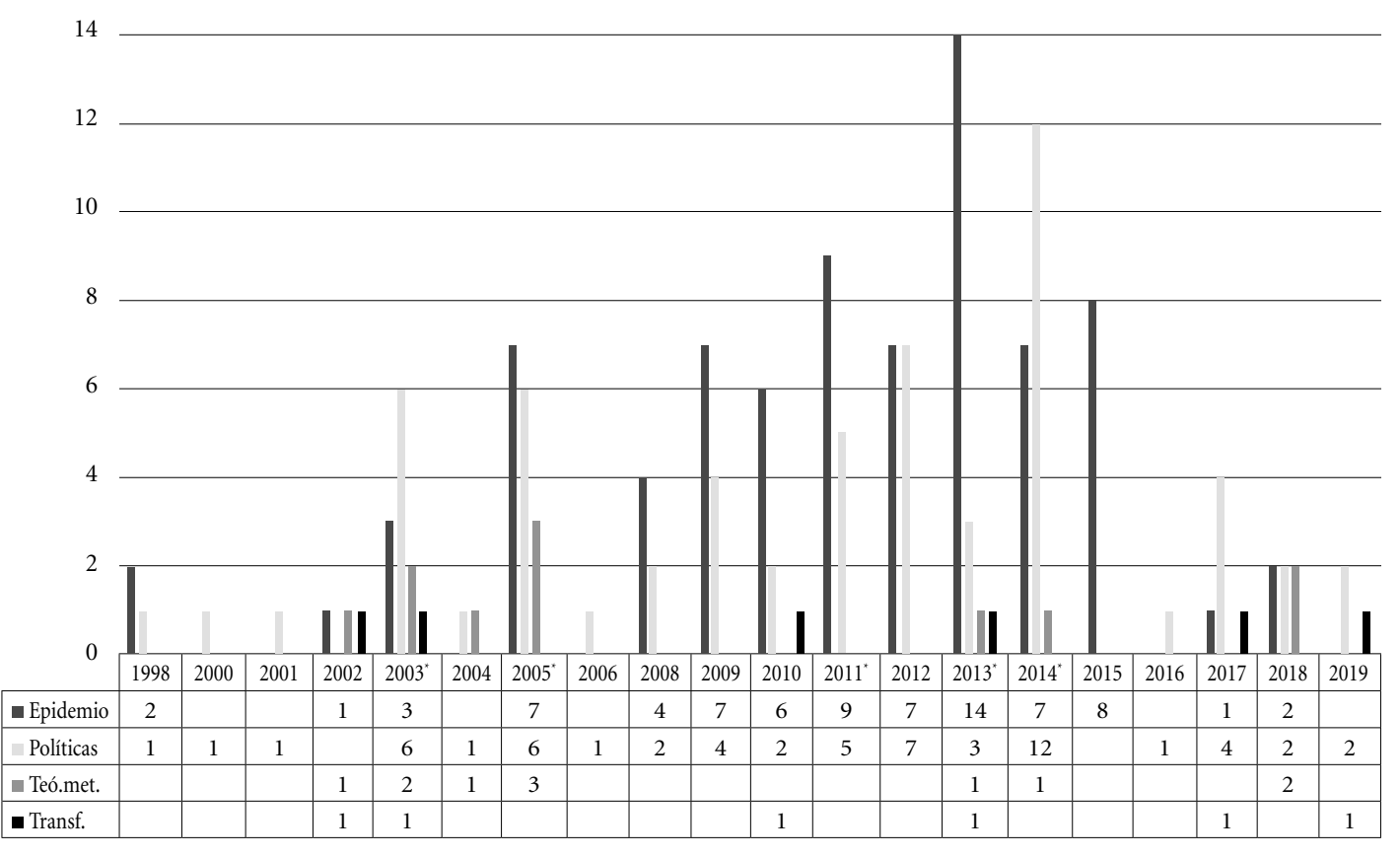

Figura 1. Distribuição das publicações em Saúde do Trabalhador na RC\&SC nos anos 1996 a 2019.

Fonte: Imagem construída a partir de formulação dos autores sobre as publicações selecionadas na RC\&SC no período de 1996 a 2019. Os anos marcados com asterisco indicam a publicação de números temáticos em ST.

Outros 13 estudos relacionaram o processo de adoecimento dos trabalhadores com a organização social e os processos de reprodução social. Estes se aproximam do referencial da epidemiologia crítica cunhada por Breilh ${ }^{26}$ que se fundamenta em uma compreensão de saúde e doença que coloca no centro da explicação da determinação da saúde, o trabalho - a produção capitalista - e, a ele subordinado, a vida - o consumo sob o comando do processo de acumulação capitalista. Assim, as diferentes formas de inserção de classe dos trabalhadores no modo de produção determinam seus perfis de reprodução social e de adoecimento

Os estudos em questão investigaram os processos saúde-doença dos trabalhadores relacionando às diferentes formas de desgaste e adoecimento com as diversas formas de inserção no processo de reprodução social de mineradores, trabalhadores da indústria, petroleiros, pesca- dores e catadores de marisco e trabalhadores da saúde.

O grupo temático Políticas Públicas e ST abarca 61 artigos a respeito de ações desenvolvidas no interior dos serviços de saúde do SUS e, destes, 22 são relativos às ações de Visat.

Dentre os assuntos abordados destaca-se a implantação da Renast e da rede regional dos CEREST e suas ações. Identifica-se a preocupação com o processo de estruturação das ações de ST articuladas aos princípios e diretrizes do SUS; com as ações de matriciamento; com a articulação das ações de ST no SUS e outros setores (Previdência, Trabalho e Ambiente); com o reconhecimento da necessidade de um sistema de informação para subsidiar o planejamento e a intervenção adequados; bem como com a relação entre produção, saúde e ambiente.

Sobre a participação social, foram identificados artigos que tratavam da formação em ST de 


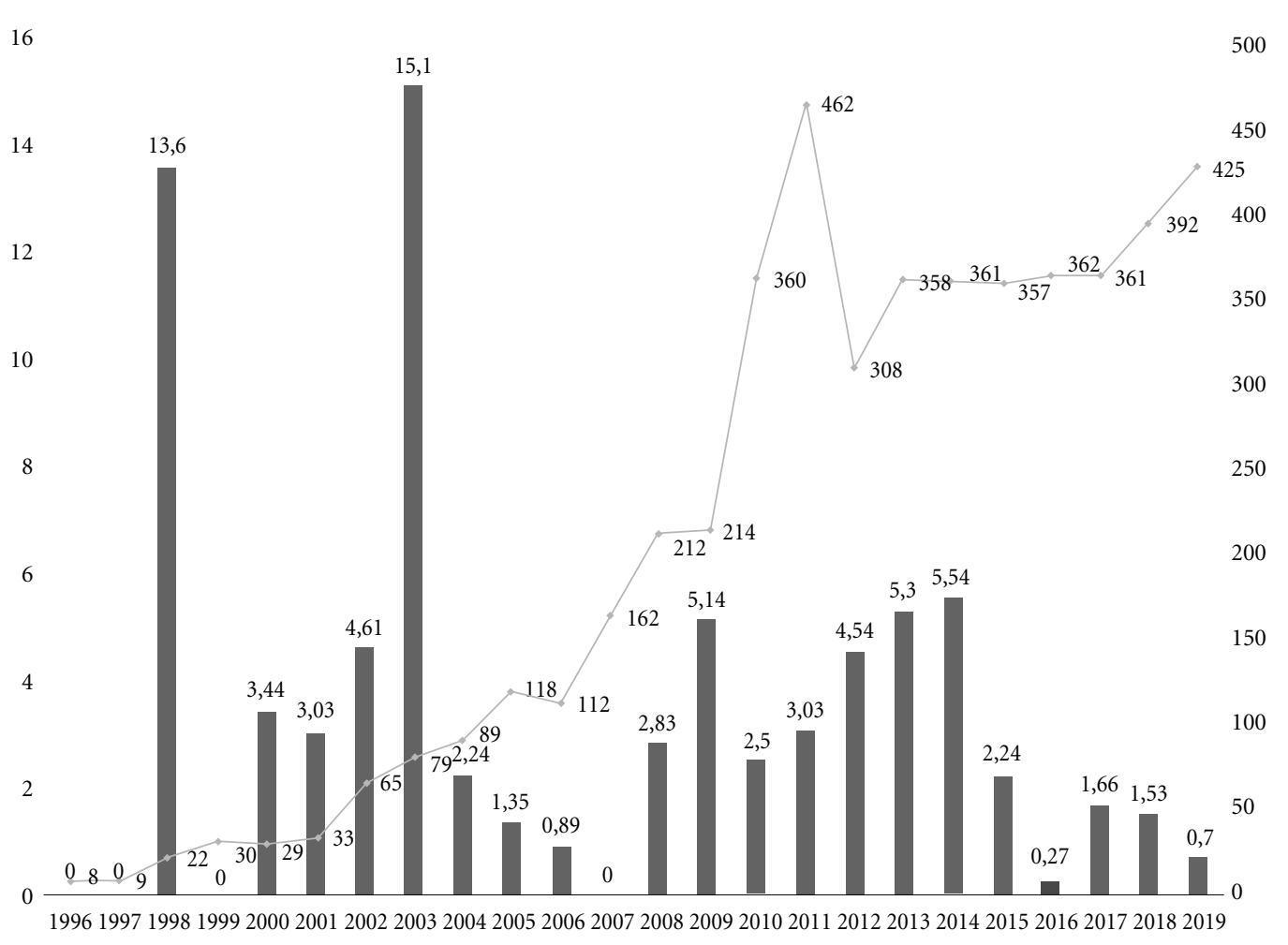

Figura 2. Comparativo entre o total de artigos publicados pela RC\&SC e o percentual dos artigos em ST diante do total nos anos 1996 a 2019.

Fonte: Imagem construída a partir de formulação dos autores sobre as publicações selecionadas na RC\&SC no período de 1996 a 2019.

diretores sindicais e também referente ao exame crítico do posicionamento de entidades sindicais com relação aos planos de saúde e ao SUS e um texto que abordou as Conferências Nacionais de Saúde do Trabalhador.

As ações de Visat configuram um tema caro ao campo ST, o que se pode apreender pelo número de artigos sobre esse assunto, os quais versam sobre: criação de instrumentos e modelos de monitoramento dos ambientes e condições de trabalho; interlocução com as vigilâncias sanitárias, epidemiológica e ambiental; ações intersetoriais, destacando-se um que envolveu a universidade e o CEREST de Piracicaba (SP) no desenvolvimento de metodologia para ações de intervenção no trabalho de corte da cana de açúcar.

No grupo temático Estudos Teórico-metodológicos os artigos mostram a diversidade de disciplinas que constituem ou dialogam com o campo ST e as abordagens epistemológicas so- bre questões do campo. Destacam-se textos que examinam a multi/inter/transdisciplinariedade para refletir sobre as relações entre disciplinas no campo ST; estudos sobre os métodos de pesquisa-intervenção a partir de algumas abordagens teórico-metodológicas; e outro, sobre a trajetória do campo ST no SUS, fazendo um balanço dos seus desafios.

O GT Transformações do Mundo do Trabalho e ST é composto de 6 artigos. Alguns enfatizam questões relacionadas às configurações macrossociais no neoliberalismo - dívida externa, globalização, crítica ao modelo de desenvolvimento que leva à pobreza, ao desemprego - buscando relacioná-las às condições do trabalho e ao ambiente. Outro grupo de artigos aborda mudanças nas formas de gestão e processos de trabalho no setor industrial. São estudos que adentram ao local de trabalho. A nova organização do trabalho docente de universidades públicas também foi 
estudada nesse GT, em sua relação com a sobrecarga, pensando-se a intensificação e a precarização do trabalho docente.

\section{Discussão}

Os artigos da C\&SC analisados abordaram o campo ST de maneira abrangente e diversificada. A produção tem sido praticamente constante a partir de 1998 - dois anos após a publicação de sua primeira edição -, ainda que haja, em seus 25 anos de existência, a ausência de artigos desse campo em 4 anos já especificados (1996, 1997, 1999 e 2007).

Os textos analisados no grupo temático "Estudos Epidemiológicos" evidenciam uma maior produção com ênfase no estabelecimento de nexos causais entre doenças e possíveis agentes etiológicos. Este resultado reflete a produção científica do campo da saúde, profundamente influenciada pela perspectiva biomédica ${ }^{27-30}$, em detrimento dos princípios teóricos da Medicina Social ${ }^{26,31}$.

De certo modo, podemos inferir que a preponderância de estudos centrados na perspectiva biomédica, frequentemente associada à publicação do Relatório Flexner, cujas diretrizes orientavam para o estudo das doenças e da ruptura com a ciência de base metafísica para a sustentação no modelo racionalista ${ }^{32}$, também se reflete no menor número de estudos sobre o mundo do trabalho e de vertente teórico-metodológica.

Ressalta-se o número reduzido de artigos voltados para as transformações do mundo do trabalho e relacionados a ST quando comparado aos estudos epidemiológicos. Os estudos tratam das mudanças nas políticas macrossociais sob o neoliberalismo globalizado e das implicações dessas transformações no cotidiano de trabalhadores com impactos na saúde destes. Considerando-se o contexto atual marcado pela reestruturação produtiva ${ }^{21}$ e suas consequências para a saúde dos trabalhadores ${ }^{33}$, essa realidade mereceria ser estudada de modo mais atento, regular e prolongado no tempo. Hoje, o trabalho informal atinge cerca de $42 \%$ da população ocupada ${ }^{34}$. Tanto a informalidade decorrente da reestruturação produtiva, como aquela que historicamente compõem o mercado de trabalho no Brasil. Tal lacuna é esperada, pois a construção do campo ocorreu como um movimento que articulou os serviços de saúde pública e os sindicatos de trabalhadores formais ${ }^{35}$. Em sua trajetória, o campo ST tem focalizado prioritariamente o trabalho assalariado, mesmo que o Brasil nunca tenha sido uma sociedade salarial, conforme aponta Castel $^{36}$. Nessa perspectiva, o desemprego, como temática relacionada à saúde, também mereceria receber maior atenção dos estudos do campo ST, para além daqueles que tratam da saúde mental e trabalho ${ }^{22}$.

O conjunto de artigos identificados como "Estudos Teórico-Metodológicos”, apresentam um pluralismo epistêmico que historicamente dão substância ao campo Saúde do Trabalhador (ST), demonstrando a coexistência de diversas matrizes teóricas, que têm orientado pesquisas e práticas neste âmbito. Entretanto, ainda são escassos os estudos conceituais e pesquisas-base, bem como a proposição de metodologias que contribuíram com o fortalecimento e desenvolvimento do campo ST ${ }^{1}$.

No grupo temático Políticas Públicas, foi observada uma quantidade considerável de estudos, cuja análise permite identificar ao menos dois movimentos, cujos princípios gerais são integrantes do mesmo processo de implantação e implementação das ações de ST no SUS. Um deles é conformado por textos que analisam, discutem e apresentam elementos da implementação da política de ST no SUS, os mecanismos e os conflitos para a sua estruturação, o desafio da intersetorialidade e o controle social. O outro é composto por trabalhos que tratam especificamente das ações de VISAT, que também envolvem a estruturação das ações, a intersetorialidade e a participação social.

No tocante à implementação da política de ST no SUS ${ }^{16}$, as publicações são representativas dos inúmeros desafios enfrentados para a sua efetivação, cujas questões centrais podem ser resumidas em: a criação de sistemas de informação de $\mathrm{ST}^{37}$, a inclusão da SMRT no contexto dos CEREST ${ }^{38}$, bem como a relação entre produção, saúde e ambiente $^{39} \mathrm{e}$, entre outros, o controle social ${ }^{40}$.

Os estudos analisados dedicados à VISAT, por sua vez, podem ser divididos em dois enfoques: 1) que trata da estruturação das ações de VISAT, considerando normatizações e metodologias criadas pelo CEREST, a incorporação das ações na Atenção Básica e dos problemas relativos à saúde mental e trabalho, como objeto de ação da VISAT $^{41}$; e 2) que trata de experiências de VISAT nas particularidades de alguns setores econômicos e/ou agentes desencadeadores de morbi-enfermidades de alguns setores econômicos.

Ressalta-se que nessa perspectiva não é possível desvincular a estruturação da VISAT das políticas públicas de saúde como um todo e os 
ataques que as políticas sociais, especialmente o SUS, vêm sofrendo, afeta também as políticas voltadas para a saúde do trabalhador ${ }^{42}$.

Sendo assim, estudos sobre a proteção social, na perspectiva da Seguridade, conforme previsto na Constituição Federal de 1988, são necessários, na medida em que as recentes contrarreformas de Estado, ao extinguirem o Ministério do Trabalho e o da Previdência Social, pautam-se pela lógica do mercado, negando direitos sociais conquistados, pois as atribuições daqueles ministérios foram assumidas pelo Ministério da Economia ${ }^{43}$.

\section{Conclusões}

Este percurso retrata uma visão panorâmica das características da produção científica, relativa ao campo da saúde do trabalhador, dentre os muitos desenhos possíveis de análise e composição dos dados, presentes no corpus constituído a partir dos artigos da C\&SC.

Ademais dos avanços constatados, como a expressiva produção de pesquisas epidemiológicas e sobre políticas públicas, os estudos analisados sinalizam elementos que se traduzem em desafios para o pleno desenvolvimento do campo ST. E originam mais perguntas que respostas.

É notório que o movimento da Reforma Sanitária foi contemporâneo ao que podemos chamar de movimento pela Saúde do Trabalhador, cabendo afirmar que estabeleceram pouca interlocução no sentido de que pudessem nutrir-se mutuamente para construir o SUS. Nesse sentido, seria interessante indagar sobre as possíveis articulações políticas entre os dois movimentos na perspectiva do fortalecimento do SUS, o que poderia constituir-se em objeto de estudos.

O controle social, tema caro ao campo, mereceria ser investigado sob perspectiva que iluminasse as dificuldades de exercê-lo, à luz da realidade atual do movimento sindical brasileiro, o qual se encontra fragilizado quando comparado ao papel tido na década de 1980 . Prado ${ }^{44}$ tece críticas ao controle social no SUS, identificando diversas dificuldades para esse exercício, na medida em que ele se reduz à participação nos conselhos de saúde. Que alternativas se abrem diante desta problemática?

No que se refere às políticas públicas e à intersetorialidade, estudos sobre a proteção social, na perspectiva da Seguridade, conforme previsto na Constituição Federal de 1988, são necessários, na medida em que as recentes contrarreformas de Estado se pautam pela lógica do mercado, negando direitos sociais conquistados.

Quais caminhos trilhar para a efetivação da Política de Seguridade Social, diante da extinção do Ministério do Trabalho e do da Previdência Social, considerando que as atribuições daqueles ministérios foram assumidas pelo Ministério da Economia? ${ }^{43}$.

Nesta perspectiva de ataque às políticas públicas, no que diz respeito ao seu financiamento no contexto das políticas neoliberais que preconizam o Estado mínimo, há sérios limites para o SUS como uma política universal, integral, igualitária e com participação social, afetando também as políticas voltadas para a saúde dos trabalhadores ${ }^{42,45}$.

As intensas transformações no mundo do trabalho e o desemprego, como temáticas relacionadas à saúde, assim como estudos que tratam especificamente da saúde mental e trabalho, recebem uma tímida atenção dos estudos do campo ST, levando-nos a questionar o porquê.

Diante do exposto, entendemos que há um longo caminho para a retomada do potencial transformador que as pesquisas e as ações dentro do SUS, relativas ao campo ST, abriram após a redemocratização do país como elementos importantes para a consolidação de Políticas Públicas voltadas para o bem-viver da população e a qualidade dos serviços de saúde que compõem o SUS.

\section{Colaboradores}

FAC Lacaz elaborou a concepção teórica, participou da discussão, redação e revisão do texto. PM Goulart, EA Souza, CA Trapé, D Moita, G MotaSousa e B Chapadeiro participaram na elaboração, discussão, redação e revisão do texto. 


\section{Referências}

1. Lacaz FAC. Saúde do Trabalhador: um estudo sobre as formações discursivas da academia, dos serviços e do movimento sindical [tese]. Campinas: Faculdade de Ciências Médicas; 1996.

2. Strausz MC, Guilam MCR, Oliveira SS. A intervenção em saúde do trabalhador na perspectiva dos atores históricos do campo. Rev Bras Saúde Ocup. 2019; 44(25):1-12.

3. Minayo-Gomez C. Campo da Saúde do Trabalhador: trajetória, configuração e transformações. In: Minayo-Gomez C, Machado JMH, Pena PGL, organizadores. Saúde Do Trabalhador Na Sociedade Brasileira Contemporânea. Rio de Janeiro: Editora Fiocruz; 2011. p. 23-34.

4. Gomez CM, Lacaz FAC. Saúde do Trabalhador: novasvelhas questões. Cien Saude Colet 2005; 10(4):797-807.

5. Lacaz FAC. O campo Saúde do Trabalhador: resgatando conhecimentos e práticas sobre as relações. Occup Health (Auckl) 2007; 23(4):757-766.

6. Mendes R, Dias EC. Da medicina do trabalho à saúde do trabalhador. Rev Saude Publica 1991; 25(5):341349.

7. Marx K. O Capital: Crítica Da Economia Política. Rio de Janeiro: Civilização Brasileira; 2006.

8. Laurell AC, Noriega M. Processo de Produção e Saúde - Trabalho e Desgaste Operário. São Paulo: Hucitec: 1989.

9. Laurell AC. A saúde-doença como processo social. In: Nunes ED, organizador. Medicina Social: Aspectos Históricos e Teóricos. São Paulo: Global; 1983. p. 133-158.

10. Laurell AC. Para La Investigación Sobre La Salud de Los Trabajadores. Washington: OPAS; 1993.

11. Oddone I, Marri G, Gloria S. Ambiente de Trabalho: A Luta Dos Trabalhadores Pela Saúde / Saúde Em Debate. São Paulo: Hucitec; 1986.

12. Rebouças AJA. Insalubridade: Morte Lenta No Trabalho. São Paulo: Diesat/Oboré; 1989.

13. Lacaz FAC. Política Nacional de saúde do trabalhador: desafios e dificuldades. In: Lourenço E, Navarro V, Silva J, Santana RS, organizadores. Avesso Do Trabalho II: Trabalho, Precarização e Saúde Do Trabalhador. São Paulo: Expressão Popular; 2010. p. 199-230.

14. Brasil. Portaria $n^{\circ} 1.823$, de 23 de agosto de 2012. Institui a Política Nacional Do Trabalhador e Da Trabalhadora. Diário Oficial da União 2012, 23 ago.

15. Vasconcellos LCF, Gomez CM, Machado JMH. The gap between what has been defined and what is still pending in occupational health surveillance. Cien Saude Colet 2014; 19(12):4617-4626.

16. Dias EC, Hoefel MG. O desafio de implementar as ações de saúde do trabalhador no SUS: a estratégia da RENAST. Cien Saude Colet 2005; 10(4):817-828.

17. Vianna LCrR, Ferreira AP, Vasconcellos LCF, Bonfatti JR, Oliveira MHB. Vigilância em Saúde do Trabalhador: um estudo à luz da Portaria ${ }^{\circ}$ 3.120/98. Saúde Debate 2017; 41(114):786-800.

18. Lacaz FADC, Trapé A, Soares CB, Santos APL. Estratégia Saúde da Família e Saúde do Trabalhador: um diálogo possível? Interface (Botucatu) 2013; 17(44):75-87.

19. Gomez CM, Vasconcellos LCF, Machado JMH. Saúde do trabalhador: aspectos históricos, avanços e desafios no Sistema Único de Saúde. Cien Saude Colet 2018; 23(6):1963-1970.
20. Lacaz FAC. Continuam a adoecer e morrer os trabalhadores: as relações, entraves e desafios para o campo Saúde do Trabalhador. Rev Bras Saude Ocup 2016; 41(13):1-11.

21. Antunes R. O Privilégio Da Servidão - o Novo Proletariado de Serviços Na Era Digital. São Paulo: Boitempo; 2018.

22. Seligmann-Silva E. Trabalho e Desgaste Mental: O Direito de Ser Dono de Si Mesmo. São Paulo: Cortez; 2011.

23. Schwab K. The Fourth Industrial Revolution. Genebra: World Economic Forum; 2016.

24. Minayo-gomez C, Thedim-Costa SMF. Incorporação das ciências sociais na produção de conhecimentos sobre trabalho e saúde. Cien Saude Colet 2003; 8(1):125-136.

25. Lima EP, Lopes SMB, Amorim MIM, Araújo LHS, Maia ER. Exposição a pesticidas e repercussão na saúde de agentes sanitaristas no Estado do Ceará, Brasil. Cien Saude Colet 2009; 14(6):2221-2230.

26. Breilh J. Epidemiología del siglo XXI y ciberespacio : repensar la teoría del poder y la determinación social de la salud. Rev Bras Epidemiol. 2015; 18(4):972-982.

27. Bennet G. The Wound and the Doctor: Healing, Technology and Power in Modern Medicine. London: Martin Secker and Warburg; 1987.

28. Barros JAC. Pensando o Processo Saúde Doença: A que responde o modelo biomédico? Saúde Soc 2002 11(1):67-84.

29. Backes MTS, Rosa LM, Fernandes GCM, Becker SG, Meirelles BHS, Santos SMA. Conceitos de Saúde e Doença ao longo da História sob o olhar epidemiológico e antropológico. Rev enferm UERJ 2009; 17(1):111-117

30. Puttini RF, Junior AP, Oliveira LR. Abordagem biopsicossocial e auto-organização. Physis 2010; 20(2):753767.

31. Breilh J, Granda E. Investigação Da Saúde Na Sociedade: Guia Pedagógico Sobre Um Novo Enfoque Do Método Epidemiológico. 2a ed. São Paulo: Cortez, Instituto de Saúde/Abrasco; 1989.

32. Fertonani HP, Pires DEP, Biff D, Scherer MDA. The health care model: Concepts and challenges for primary health care in Brazil. Cien Saude Colet 2015; 20(6):1869-1878

33. Lourenço EAS, Lacaz FAC, Goulart PM. Crise do capital e o desmonte da Previdência Social no Brasil. Serv Soc Soc 2017; (130):467-486.

34. Fundação Getúlio Vargas, Instituto Brasileiro de Economia. Boletim Macro 2019. [Boletim na Internet] 2019. [acessado 2020 Abr 28]. Disponível em: nhttps://portalibre.fgv.br/data/files/58/37/B3/E5/5B7AE610B0BBB6E68904CBA8/BoletimMacroIbre_1911. pdf.

35. Sato L, Lacaz FAC, Bernardo MH. Psychology and the Workers' Health Movement in the State of São Paulo (Brazil). J Health Psychol 2004; 9(1):121-130.

36. Castel R. As Metamorfoses Da Questão Social: Uma Crônica Do Salário. Petrópolis: Vozes; 1995.

37. Facchini LA, Nobre LCC, Faria NMX, Fassa AG, Thumé E, Tomasi E, Santana V. Sistema de Informação em Saúde do Trabalhador: desafios e perspectivas para o SUS. Cien Saude Colet 2005; 10(4):857-867. 
38. Sato L, Bernardo MH. Saúde Mental e Trabalho: os problemas que persistem. Cien Saude Colet 2005; 10(4):869-878.

39. Dias EC, Rigotto RM, Augusto LGS, Cancio J, Hoefel MGL. Saúde ambiental e saúde do trabalhador na atenção primária à saúde no SUS: oportunidades e desafios. Cien Saude Colet 2009; 14(6):2061-2070.

40. Lacaz FAC, Flório SMR. Controle social, mundo do trabalho e as Conferências Nacionais de Saúde da virada do século XX. Cien Saude Colet 2009; 14(6):21232134.

41. Araújo TM, Palma TDF, Araújo NC. Vigilância em Saúde Mental e Trabalho no Brasil: características , dificuldades e desafios. Cien Saude Colet 2017; 22(10):3235-3246.

42. Lourenço E, Goulart PM, Anunciação L, Lacaz FAC. Condições de trabalho de assistentes sociais da área da saúde e repercussões psicossociais. Saúde Soc 2019; 28(1):154-168.

43. Brasil. Medida Provisória no 870 , de 1 de janeiro de 2019. Estabelece a organização básica dos órgãos da Presidência da República e dos ministérios. Diário Oficial da União 2019; 1 jan.

44. Prado E. Controle social no SUS é sinônimo de conselho de saúde. Ens e Dialog em Saude Colet 2017; (5):20-22.

45. Associação Brasileira de Saúde Coletiva (Abrasco). Manifesto em defesa do SUS - "Saúde e Democracia: desafios para o Brasil contemporâneo". [Notícia na Internet] 2018 [acessado 2020 Abr 27]. Disponível em: https://www.abrasco.org.br/site/noticias/movimentos-sociais/manifesto-em-defesa-do-sus-saude -e-democracia-desafios-para-o-brasil-contemporaneo/33154/.

Artigo apresentado em 30/04/2020

Aprovado em 22/06/2020

Versão final apresentada em 24/06/2020 